\title{
Thyroid Papillary Carcinoma and Noninvasive Follicular Thyroid Neoplasm with Papillary-Like Nuclear Features (NIFTP)
}

\author{
Mehmet Celik* \\ Department of Internal Medicine and Endocrinology and Metabolism, Trakya University, Turkey \\ *Corresponding author: Mehmet Celik, Department of Internal Medicine and Endocrinology and Metabolism, Trakya University, Turkey \\ Submission: 海 August 22, 2017; Published: 海 October 23, 2017
}

\section{Editorial}

Papillary thyroid carcinomas (PTC) are well differentiated malignante pithelial tumors with characteristic nuclear features and they originate from epithelial cells of thyroid follicle. Papillary thyroid carcinoma is the most common type of thyroid cancers, which composes $85-90 \%$ of all thyroid carcinomas. Number of patients diagnosed with thyroid cancer has significantly increased during the last two decades due to increased awareness of nodular thyroid diseases, developments in diagnostic methods, wide applicability of thyroid fine needle aspiration, new descriptions of histopathology criteria and increased radiation exposure. It is more common in males than females with some ethnic variations. Although it is very rare during early childhood, it is the most common thyroid cancer of this age group. The mean age is 46 years at the time of diagnosis. Tumor has some histologic variants, the most common ones are being classical and follicular variants.

Although PTCs generally show good prognostic course with indolent features. Some PTCs may demonstrate bad prognostic course. Prognosis is closely related to disease stage; 10 -year survival is $99,8 \%$ and $40,7 \%$ in stage I and stage IV disease, respectively. 7th edition of AJCC TNM Classification System of Malignant Tumors (2010) reported size of primary tumor $(>2 \mathrm{~cm})$, extra thyroidal extension, distant metastasis, lymphnode metastasis and age at the time of diagnosis (above 45 years) as the most important criteria in evaluating biological features of these tumors. In addition, multi focality, vascular invasion, in complete surgery, some specific variants and male gender have been suggested as potential prognostic factors [1-6].

Some histologic variants of these tumors are known to show aggressive behavior. In recent years, the frequency of follicular variant papillary thyroid cancer (FVPTC) has been shown to increase in frequency among these tumors and since FVPTCs not always have nuclear features of classical variant papillary carcinoma and some of them may show quite aggressive clinical behavior, debates on their diagnostic and prognostic features take an important place in recent studies. Moreover, some molecular features of FVPTCs are different than classical variant PTC; they mostly demonstrate mutations as seen in follicular adenoma or follicular carcinoma. These types of tumors mostly show RAS mutations, while they rarely have BRAF mutations. Although the term "well differentiated tumor with unknown malignan cypotential" acquired currency for these tumors after 2000s, this description has led to some uncertainty in clinical management and therefore has not been approved. Some studies on this topic suggested that rather than nuclear features, capsule and vascular invasion were more important determinants in clinical behavior [7]. However, some other studies reported aggressive behavior in tumors with macro follicular hyper plastic nodular paternal though they partly have nuclear features of classical variant papillary carcinoma [8]. As suggested in the study by Can et al. [6], FVPTCs are more common than classical variant tumors of PTCs and they pose problems in clinical management of these patients while choosing appropriate treatment modality (RAI or complementary surgery/lymphnoded is section), since these approaches have many negative effects on health spending and psychological states of patients.

Recent studies have shown that about $10-20 \%$ of all thyroid cancers is composed of non-invasive capsulated papillary follicular variant (NIFVPTC) $[9,10]$. However, since there is no consensus on papillary nuclear changes in the diagnosis of NIFVPTK, there is difference of opinion. In addition, a group of specialist has recently suggested the term "Noninvasive follicular thyroid neoplasm with papillary like nuclear features (NIFTP)" instead of NIFVPTC [11]. Although NIFPT is less aggressive, whether these patients are exposed to unnecessary treatment and follow-up procedures is a recent question of debate. Contribution of clinical and laboratory parameters used in the evaluation of thyroid nodules to the diagnosis of PTC in filtrative follicular variant and NIFTP is still unknown. Clinico pathological criteria NIFTP; tumors exhibiting dominant follicular architecture ( $\leq 1 \%$ true papillae) and nuclear features of papillary thyroid carcinoma (2-3/3 nuclear score according to the 
alterations in nuclear size and shape; nuclear overlapping, nuclear enlargement, nuclear memsbrane irregularities; irregular nuclear contours, nuclear elongation, intra nuclear pseudo inclusions, nuclear grooves, chromatin characteristics; nuclear chromatin clearing, glassy nuclei, peripheral margination of the chromatin) but not associated with necrosis, solid/trabecular/cribriform growth patterns, tall cell or columnar cell cyto morphology or more than 3 mitoses per 10 high power field (HPF) were classified as FVPTC [11].

In the literature, only 2 subjects $(0.6 \%)$ have been shown to have recurrence among 352 well-documented noninvasive FVPTC. One of these subjects had under gone in complete excision and non invasive nature of tumor was questionable in other patient. In general, data suggest that the rate of negative result is very low in the absence of invasion of this lesion [12-18].

\section{Conclusion}

In conclusion, further studies are required to evaluate long term results of the patients with clinic opathologic criteria of NIFTP and NIFTP should be classified in the group of low risk papillary thyroid cancer despite low rate of negative results.

\section{References}

1. Aschebrook-Kilfoy B, Ward MH, Sabra MM, Devesa SS (2011) Thyroid cancer incidence patterns in the United States by histologic type, 19922006. Thyroid 21(2):125-134.

2. Haugen BR, Alexander EK, Bible KC, Doherty GM, Mandel SJ, eta l. (2015) American Thyroid Association Management Guidelines for Adult Patients with Thyroid Nodules and Differentiated Thyroid Cancer: The American Thyroid Association Guidelines Task Force on Thyroid Nodules and Differentiated Thyroid Cancer. Thyroid 26(1): 1-133.

3. Can N, Ayturk S, Celik M, Sezer YA, Ozyilmaz F, et al. (2016) Histological perspective on the effects of tumor-associated macrophages in the tumor micro environment surrounding papillary thyroid carcinoma. Pol J Pathol 67(4): 332-344.

4. Lang BHH, Lo CY, Chan WF, Lam KY, Wan KY (2007) Staging Systems for Papillary Thyroid Carcinoma: A Review and Comparison. Ann Surg 245(3): 366-378

5. Sezer A, Celik M, YilmazBulbul B, Can N, Tastekin E, et al. (2017) Relationship between lympho vascular in vasion and clinic pathological features of papillary thyroid carcinoma. Bosn J Basic Med Sci 17(2): 144151.

6. Can N, Tastekin E, Ozyilmaz F, Sezer YA, Guldiken S, et al. (2015) Histopathological Evidence of Lymph Node Metastasis in Papillary Thyroid Carcinoma. Endocr Pathol 26(3): 218-228.
7. In vasion rather than nuclear features correlates without come in encapsulated follicular tumors: further evidence for there classification of the encapsulated papillary thyroid carcinoma follicular variant. Ian Ganly, Laura Wang R, Michael Tuttle B, Nora Katabi, Gustavo A, Ceballos, Ruben Harach H, Ronald Ghossein. Human Pathology (2015) 46: 657664 .

8. Lloyd RV, Erickson LA, Casey MB, Lam KY, Lohse CM, et al. (2004) Observer variation in the diagnosis of foliccular variant of papillary thyroid carcinoma. Am J Surg Pathol 28(10): 1336-1340.

9. Jung CK, Little MP, Lubin JH, Brenner AV, Wells SA, et al. The increase in thyroid cancer incidence during the last four decades is accompanied by a high frequency of BRAF mutations and a sharp increase in RAS mutations. J Clin Endocrinol Metab 99(2): E276-E285.

10. Lupi C, Giannini R, Ugolini C, Proietti A, Berti P, et al. Association of BRAF V600E mutation with poor clinic opathological outcomes in 500 consecutive cases of papillary thyroid carcinoma. J Clin Endocrinol Metab 92(11): 4085-4090.

11. Nikiforov YE, Seethala RR, Tallini G, Baloch ZW, Basolo F, et al. (2016) Nomenclature revision for encapsulated follicular variant of papillary thyroid carcinoma: a paradigm shift to reduce over treatment of indolenttumors. JAMA Oncol 2(8): 1023-1029.

12. Vivero M, Kraft S, Barletta JA (2013) Risk stratification of follicular variant of papillary thyroid carcinoma. Thyroid 23(3): 273-279.

13. Piana S, Frasoldati A, DiFelice E, Gardini G, Tallini G, et al. (2010) Encapsulatedwell-differentiatedfollicular-patternedthyroidcarcinomas do not play a significant role in thefatalityratesfromthyroidcarcinoma. Am J Surg Pathol 34(6): 868-872.

14. Rosario PW, Penna GC, Calsolari MR (2014) Noninvasive encapsulated follicular variant of papillary thyroid carcinoma: is lobectomy sufficient for tumours $\geq 1 \mathrm{~cm}$ ? Clin Endocrinol (Oxf) 81(4): 630-632.

15. Howitt BE, Paulson VA, Barletta JA (2015) Absence of BRAF V600E in noninfiltrative, non-invasivefollicularvariant of papillarythyroidcarcinoma. Histopathology 67(4): 579-582.

16. Ganly I, Wang L, Tuttle RM, Katabi N, Ceballos GA, et al. (2015) Invasion rather than nuclear features correlates without come in encapsulated follicular tumors: further evidence for the reclassification of the encapsulated papillary thyroid carcinoma follicular variant. Hum Pathol 46(5): 657-664.

17. Baloch ZW, Li Volsi VA (2000) Encapsulated follicular variant of papillary thyroid carcinoma with bone metastases. Mod Pathol 13(8): 861-865.

18. Can N, Celik M, Sezer YA, Ozyilmaz F, Ayturk S, et al. (2017) Follicular morphological characteristics may be associated within vasion infollicular thyroid neoplasms with papillary-like nuclear features. Bosn J Basic Med Sci 17(3): 211-220. 